\title{
Atypical Fibroxanthoma of the Bulbar Conjunctiva: A Unique Case Describing the Pathology, Clinical Presentation, and Management
}

\author{
Justin Kuiper Ankit Shah Rebecca Kuennen Lynn Schoenfield \\ The Ohio State University Department of Ophthalmology, Columbus, OH, USA
}

\section{Established Facts}

- Atypical fibroxanthoma is an uncommon tumor of the ocular surface with pathology revealing a highly cellular lesion with pleomorphic spindle cells, giant cells, and increased mitoses.

\section{Novel Insights}

- We provide a novel case report of atypical fibroxanthoma detailing the clinical presentation, management, and pathology and immunohistochemistry, which contributes significantly to the limited literature on this rare tumor.

\section{Keywords}

Conjunctiva · Pathology · Immunohistochemistry · Tumor · Biopsy · Fibroxanthoma

\begin{abstract}
Background/Aims: Atypical fibroxanthoma is an uncommon tumor that usually occurs in the skin of the head and neck of the elderly with significant sun exposure. We describe a unique case featuring a rare ocular surface conjunctival tumor (atypical fibroxanthoma) and provide insight on its characteristic clinical features, surgical management, and histology. Methods: A 71-year-old male fisherman with no pertinent ocular history presented to an academic center with a rapidly enlarging bulbar conjunctival mass in the right perilimbal region for the past several months. The patient underwent surgical excisional biopsy with cryotherapy, adjuvant alcohol, and amniotic membrane transplantation. Results: Pathology specimen illustrated an atypical spindle cell
\end{abstract}

\section{KARGER}

(C) 2017 S. Karger AG, Basel

E-Mail karger@karger.com

www.karger.com/oop tumor with inflammatory cells, increased mitotic activity, cytologic atypia, and positive diffuse staining with CD163 and CD10 consistent with an atypical fibroxanthoma. Conclusion: Atypical fibroxanthoma is an extremely rare ocular surface tumor that may simulate conjunctival or ocular surface squamous neoplasia. While this lesion typically pursues a benign clinical course, it may recur or rarely metastasize. Thus it should be treated aggressively with excisional biopsy, cryotherapy, absolute alcohol, and/or amniotic membrane transplantation.

(c) 2017 S. Karger AG, Basel

\section{Introduction}

Atypical fibroxanthoma is an uncommon tumor that usually occurs in the skin of the head and neck of the elderly with significant sun exposure. There have been rare reports of involvement of the ocular surface. Clinically it 

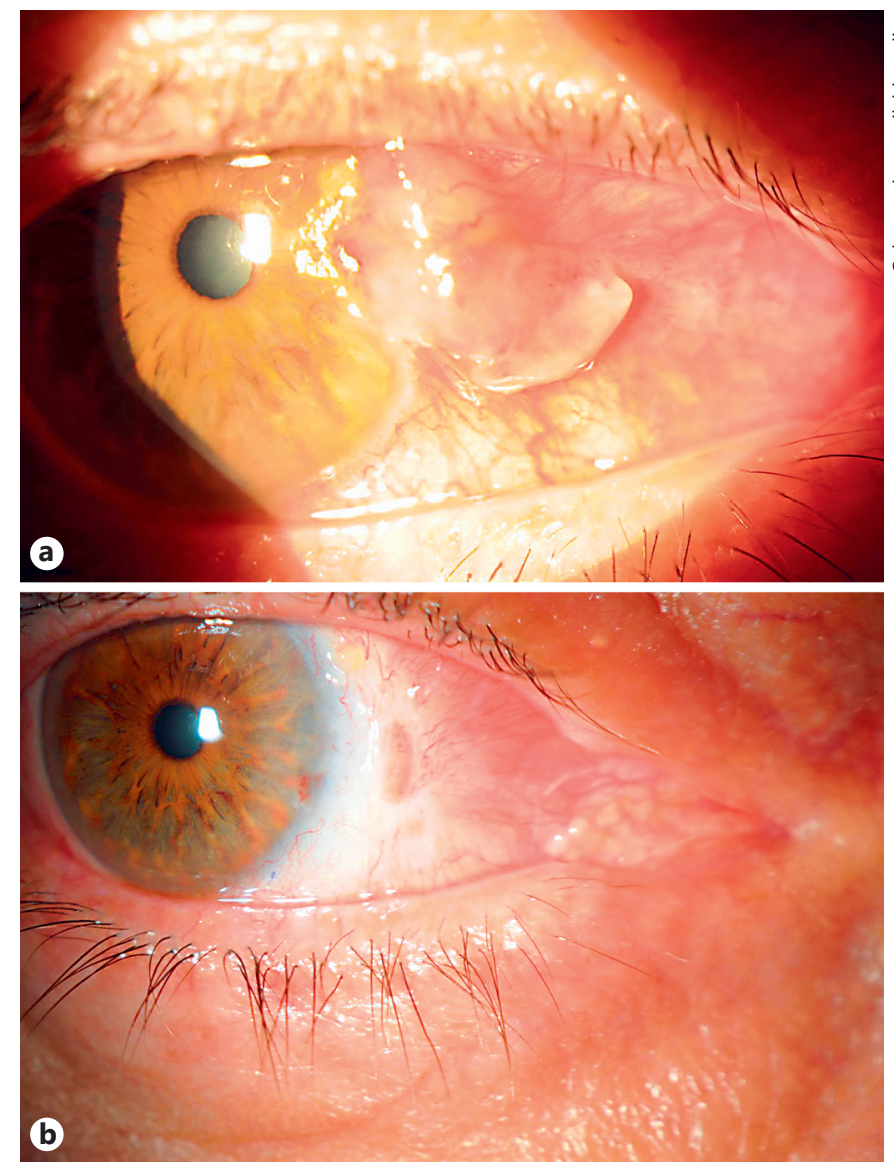

Fig. 1. a Preoperative photo demonstrating raised vascular lesion extending from the bulbar conjunctiva over the limbus. b Postoperative photo following excisional biopsy of the lesion.

may appear similar to other ocular surface tumors including melanoma, sarcoma, atypical fibrous histiocytoma, and squamous cell carcinoma [1]. Pathology reveals a highly cellular lesion with pleomorphic spindle cells, giant cells, and increased mitoses [2]. Diagnosis of the lesion requires clinical, pathological, and immunohistochemical correlation. We present a case of atypical fibroxanthoma of the bulbar conjunctiva.

\section{Case Report}

A healthy 71-year-old male with a history of hypertension, hyperlipidemia, and hypothyroidism presented to a university academic practice for evaluation of a conjunctival lesion of the right eye. The patient reported a 4-month history of a nasal conjunctival lesion that had rapidly enlarged over the past several weeks causing pain, irritation, redness, and preventing adequate closure of the eyelid. The patient denied any disturbance to his visual acuity, vi-
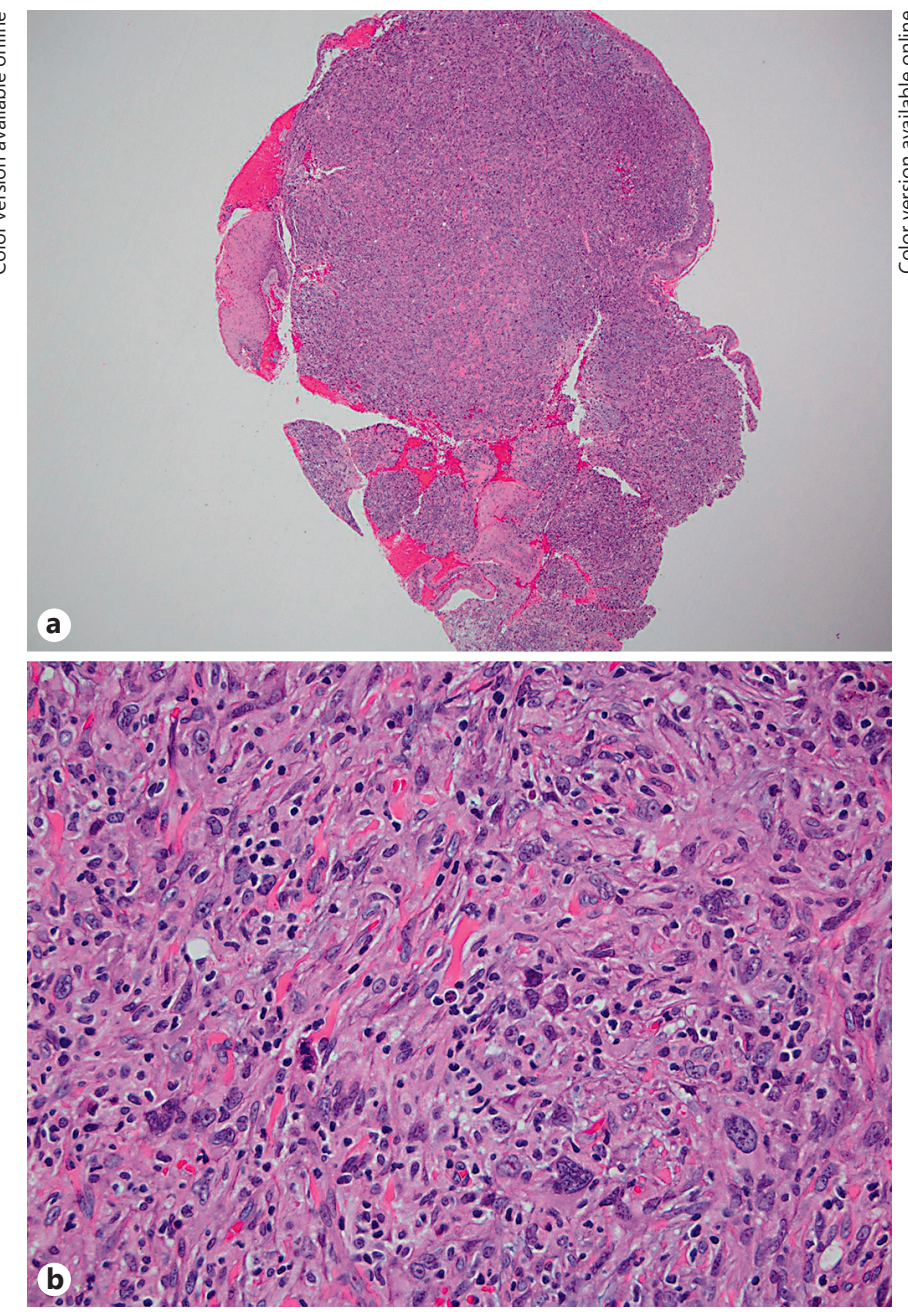

Fig. 2. a Low-power photomicrograph showing a "pyogenic granuloma"-like architecture with elevated well-circumscribed mass showing an epithelial collar and a partially ulcerated surface. Hematoxylin and eosin, 40× magnification. b High-power photomicrograph demonstrating a spindle cell population of atypical cells, some with very bizarre nuclei with multinucleation. Mitoses are present. Hematoxylin and eosin, 400× magnification.

sual field, or any bleeding from the lesion. His ocular history was significant for mild myopia and astigmatism, and he denied any previous ocular surgery. He was not using any ophthalmic medications. His family history was unremarkable. The patient worked as a fisherman in Mexico and thus had significant UV light exposure growing up.

The patient's ocular exam showed normal visual acuity, intraocular pressures, and pupillary examination in both eyes. On external examination, there was a large, pedunculated, injected lesion on the nasal perilimbal bulbar conjunctiva with fibrovascular fronds and translucent yellow nodules. The lesion measured approximately 6-7 $\mathrm{mm}$ in length without associated ulceration or pigmentation (Fig. 1a). The anterior segment and fundus exami- 

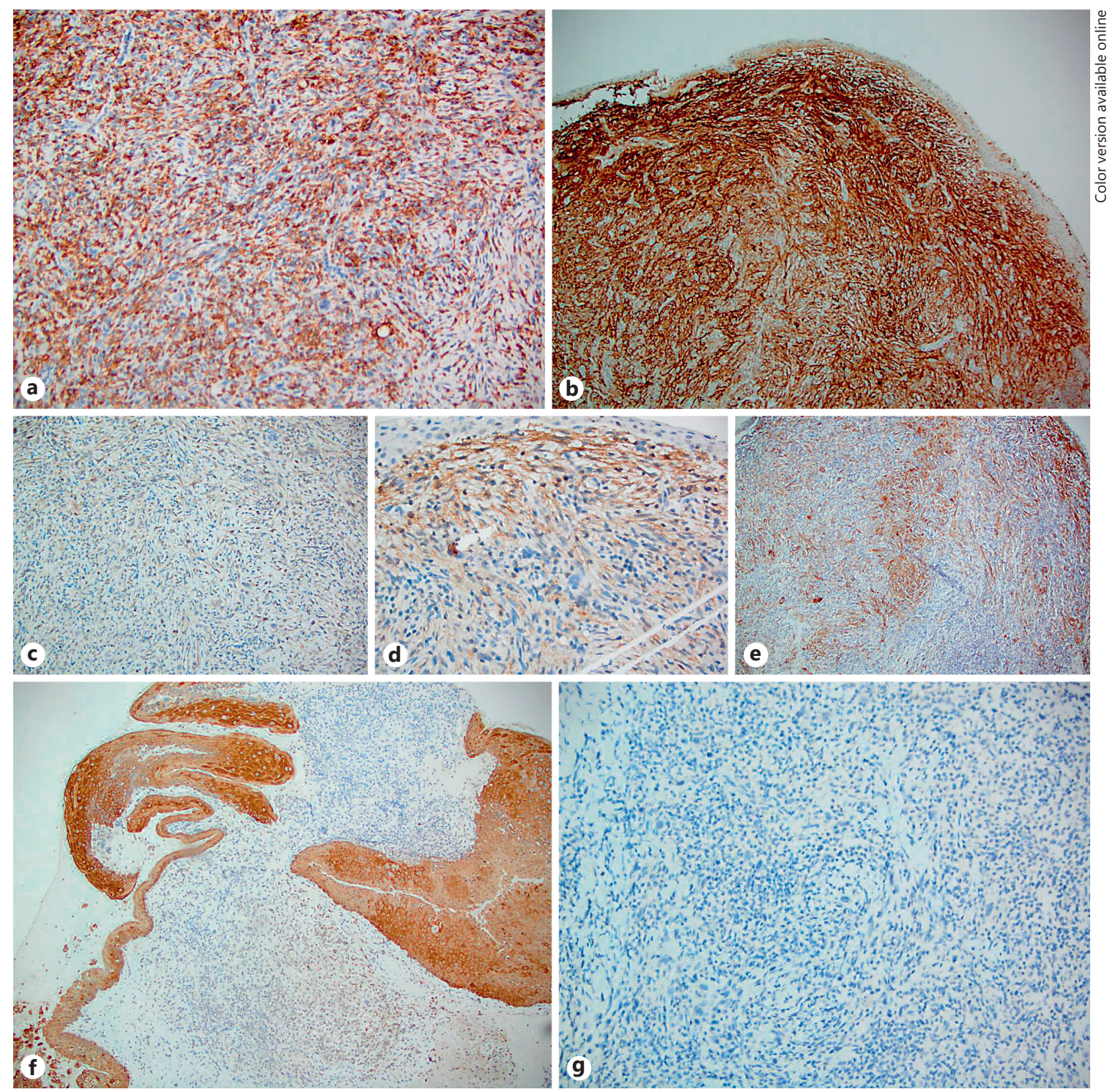

Fig. 3. Immunohistochemical profile demonstrating diffusely positive staining for CD163 (a) and CD10 (b); faint or focal positivity for calponin (c), CD99 (d), and SMA (e); and negative staining for SOX10 (f) and AE1/3 (g).

nations of the right eye and left eye were otherwise unremarkable. A decision was made to intervene surgically and the patient was scheduled for excisional biopsy of the lesion with frozen sections, cryotherapy, and ETOH. Prior to the date of surgery, the patient presented again in clinic and stated that the lesion had self-excised and was bleeding. Examination confirmed a much smaller lesion with residual large elevated stump.

The patient underwent uneventful excisional biopsy of the conjunctival lesion of the left eye with frozen sections, absolute alcohol, and cryotherapy with amniotic membrane grafting. There was no recurrence noted at the 1-month follow-up visit (Fig. 1b). Pathology revealed a proliferative spindle cell lesion (diffusely positive for CD163 and CD10 and negative for AE1/3, S100, SOX10, melanA, and CD34) admixed with inflammatory cells with in- creased mitotic activity and cytologic atypia (Fig. 2, 3). There was faint or focal positivity for CD99, smooth muscle actin (SMA), and calponin (Fig. 3). The findings are consistent with atypical fibroxanthoma.

\section{Discussion}

Atypical fibroxanthoma is an uncommon cutaneous tumor that often occurs on the head and neck region of elderly patients with extensive ultraviolet sunlight exposure $[1,3]$. The lesion may itself present as a solitary or 
multifocal red or pink dome-shaped nodule with high vascularity $[2,4]$. Histologically, it is composed of pleomorphic spindle cells with hyperchromatic nuclei and abundant cytoplasm. Despite its malignant histologic features, it usually behaves in a benign fashion with only rare cases of metastasis. Recurrences have been noted, usually when incompletely excised [5]. Risk factors for this tumor include ultraviolet and radiation exposure. Hereditary skin conditions including xeroderma pigmentosum also increase the risk of developing an atypical fibroxanthoma [6]. There have only been a few reported cases of ocular surface presentation of this tumor, and only one has been reported in patients without xeroderma pigmentosum. Other reported locations of ocular atypical fibroxanthoma include the palpebral conjunctiva, limbus, and medial canthus $[4,7,8]$.

The diagnosis of atypical fibroxanthoma is impossible to discern based solely on clinical presentation and requires histopathologic assessment along with the appropriate immunohistochemical profile [9]. Specifically, in the diagnosis of atypical fibroxanthoma, a negative profile is as important as a positive one to exclude other diagnostic possibilities such as squamous cell cancer and malignant melanoma. Atypical fibroxanthoma is negative for S100 and other melanoma markers, negative for cytokeratins (and specific markers for squamous cell differentiation), and generally negative for desmin and other muscle markers. Immunohistochemical markers that are typically positive are CD68 and CD163, alpha1-antitrypsin, alpha1-antichymotrypsin (for histiocytic lineage), as well as CD10 and CD99 [10]. Our patient's immunohistochemical profile paralleled that of most cases with positive CD68, CD163, and CD 99 stains and negative or weak S100, SOX10, AE1/3, SMA, calponin, and CD34 stains.

The rarity of this tumor on the conjunctival surface has precluded development of a standard treatment regimen; however, there is a general consensus that complete excision of the tumor yields the best prognostic outcome and lowest recurrence rate [11]. For instance, the management of dermatologic variants of fibroxanthoma includes wide local excision and Mohs microscopic surgery, both very successful but Mohs microscopic surgery producing better cosmetic result with less tissue loss [12]. In ocular management of conjunctival lesions, both bulbar and palpebral conjunctiva are included in complete excisional biopsy. Given the uncertainty of the clinical diagnosis and the difficulty in distinguishing this from aggressive malignant lesions such as squamous cell carcinoma and malignant melanoma, we recommend conservative exci- sional biopsy including frozen sections, cryotherapy, and if any evidence of corneal involvement, the use of absolute alcohol. Amniotic membrane placement using absorbable sutures is an effective measure to fill the conjunctival void. Routine postoperative care using ophthalmic antibiotic/steroid drops and close outpatient follow-up with external slit-lamp photo is also recommended.

Recurrence of atypical fibroxanthoma after excision has been reported in the dermatologic literature at 6.9$16 \%$ [13]. Data on recurrence in ocular lesions is limited, however. There are a few case reports of recurrent atypical fibroxanthoma of the eyelid and limbus $[14,15]$. Follow-up is recommended annually for monitoring of recurrence.

\section{Conclusion}

Atypical fibroxanthoma of the bulbar conjunctiva is an extremely rare tumor that often requires histopathologic evaluation with a confirmatory immunohistochemical profile. While the prognosis for conjunctival involvement is good, aggressive surgical intervention and close followup is recommended.

\section{Statement of Ethics}

Consent was obtained from the subject for the use of this case and its images in publication. No IRB approval was required for publication of this case report.

\section{Disclosure Statement}

The authors have no conflicts of interest to disclose.

\section{References}

Ocul Oncol Pathol 2018;4:152-156 DOI: $10.1159 / 000480088$
1 Lopez L, Velez R: Atypical fibroxanthoma. Arch Pathol Lab Med 2016;140:376-379.

2 Luzar B, Calonje E: Morphological and immunohistochemical characteristics of atypical fibroxanthoma with a special emphasis on potential diagnostic pitfalls: a review. J Cutan Pathol 2010;37:301-309.

3 Hsu CK, Chao SC, Shieh SJ, Lee JYY: Atypical fibroxanthoma-like amelanotic malignant melanoma: a case report and literature review. Dermatologica Sinica 2013;31:140-144.

4 Mochizuki K, Yamada T, Mori Y, Sawada A, Mori I, Ohnishi Y: Case of atypical fibroxanthoma in the palpebral conjunctiva. Jpn J Ophthalmol 2008;52:404-406. 
5 Shieh C, Daluvoy MC, Ellington KS, Praia AD: Atypical fibroxanthoma of the bulbar conjunctiva. Cornea 2015;34:1620-1622.

6 Shalabi N, Galor A, Dubovy SR, Thompson J, Bermudez-Magner JA, Karp CL: Atypical fibroxanthoma of the conjunctiva in xeroderma pigmentosum. Ocul Oncol Pathol 2015;1: 254-258.

7 Rathore D, Mohyudin MN, Mehta P, Ahluwalia HS: Atypical fibroxanthoma of the medial canthus: a rare presentation. Orbit 2013; 23:194-196.

8 Engelbrecht NE, Ford JG, White WL: Combined intraepithelial squamous neoplasia and atypical fibroxanthoma of the cornea and limbus. Am J Ophthalmol 2000;129:94-96.
9 Pujani M, Hassan MJ, Jetley S: Atypical fibroxanthoma in a young female misdiagnosed clinically as a malignant melanoma - an unusual presentation. J Cancer Res Ther 2015; 11:1027.

10 Brau Javier CN, Valentin Colon DC, Sanchez JL, Sanchec JE: Clear-cell atypical fibroxanthoma: a combined immunohistochemistry analysis. Am J Dermatopathol 2016;38:7759.

11 Shao L, Newell B, Quintanilla N: Atypical fibroxanthoma and squamous cell carcinoma of the conjunctiva in xeroderma pigmentosum. Pediatr Dev Pathol 2007;10:149-152.
12 Koch M, Freundl AJ, Agaimy A, Kiesevetter F, Kunzel J, Cicha I, Alexiou C: Atypical fibroxanthoma - histologic diagnosis, immunohistochemical markers and concepts of therapy. Anticancer Res 2015;35:5717-5735.

13 Iorizzo LJ III, Brown MD: Atypical fibroxanthoma: a review of the literature. Dermatol Surg 2011;37:146-157.

14 Paramanathan N, Gal A, Benger R: Paraffin histology sections and delayed repair in recurrent atypical fibroxanthoma of the eyelid. Orbit 2009;6:380-382.

15 Ullrich K, Wells J, Brennan C, Craig J: Recurrent atypical fibroxanthoma of the limbus. BMJ Case Rep 2013, DOI: 10.1136/bcr-2013008762. 